\title{
Pratiques
}

Linguistique, littérature, didactique

\section{Pour une approche linguistique de la poésie contemporaine : à la rencontre de James Sacré et d'Ariane Dreyfus}

For a linguistic approach to contemporary poetry: reading James Sacré and Ariane Dreyfus

\section{Michèle Monte}

\section{CpenEdition}

\section{Journals}

Édition électronique

URL : http://journals.openedition.org/pratiques/5047

DOI : $10.4000 /$ pratiques. 5047

ISSN : 2425-2042

Éditeur

Centre de recherche sur les médiations (CREM)

Référence électronique

Michèle Monte, « Pour une approche linguistique de la poésie contemporaine : à la rencontre de James Sacré et d'Ariane Dreyfus », Pratiques [En ligne], 179-180 | 2018, mis en ligne le 31 décembre 2018, consulté le 30 avril 2019. URL : http://journals.openedition.org/pratiques/5047 ; DOI : 10.4000/ pratiques. 5047

Ce document a été généré automatiquement le 30 avril 2019

(c) Tous droits réservés 


\section{Pour une approche linguistique de la poésie contemporaine : à la rencontre de James Sacré et d'Ariane Dreyfus}

For a linguistic approach to contemporary poetry: reading James Sacré and Ariane Dreyfus

Michèle Monte

1 Mon travail sur la poésie s'efforce depuis un certain nombre d'années de scruter la poésie post-1945 avec les outils de la sémantique textuelle, de la linguistique énonciative et de l'analyse de discours ${ }^{1}$. Je me propose tout d'abord de retracer brièvement les enjeux épistémologiques de ce travail, puis de mettre les outils que j'utilise à l'épreuve de deux poèmes.

\section{Bref parcours théorique}

2 Défendre une approche linguistique de la poésie ne va pas sans risques. En effet, la poésie étant perçue au sein des textes littéraires comme le lieu où la participation active du lecteur est la plus essentielle, toute tentative d'objectivation telle que la présuppose la démarche linguistique peut être accusée de réduire le texte à ses composantes matérielles en perdant de vue l'échange entre le texte et le lecteur qui, seul, pourra donner sens aux mots écrits sur la page. Reprenant le sonnet Les Chats précédemment analysé par R. Jakobson et C. Lévi-Strauss (1962) dans une analyse structurale célèbre - et controversée -, M. Riffaterre écrit en 1966 :

Comment pouvons-nous passer de la description au jugement - autrement dit de l'étude d'un texte à celle de ses effets sur le lecteur? [...] Ne peut-on supposer que le poème peut contenir certaines structures qui ne jouent pas de rôle dans sa fonction et son effet comme œuvre littéraire et que la linguistique structurale n'est peutêtre pas à même de distinguer ces structures non marquées des structures 
littérairement actives? Inversement ne négligera-t-on pas des structures exclusivement poétiques en utilisant une procédure inadaptée à la spécificité du langage poétique? (Riffaterre, 1971, p. 310)

\section{Approche énonciative et éthos de l'énonciateur textuel}

3 À la fin des années 1990 , je me suis détournée dans un premier temps des analyses structurales pour explorer ce que l'approche énonciative pouvait apporter à la compréhension de la poésie. J'étais en effet frappée par l'écart entre les recherches sur la prose narrative qui faisaient une part importante à la question des points de vue et des voix (voir par exemple Rabatel, 1998) et celles sur le poème qui faisaient peu de cas de ces questions, alors qu'en théorie littéraire la question du sujet lyrique avait déjà été posée par K. Hamburger en 1977 (traduction française en 1986) et avait fait l'objet de plusieurs colloques. Il me semblait aussi qu'en poésie lyrique au moins, l'approche pragmatique par les actes de langage pouvait être féconde, dans la mesure où le locuteur du poème, en symbiose avec l'énonciateur textuel ${ }^{2}$, se présente comme une personne engagée dans une interaction soit avec des destinataires intratextuels soit avec le lecteur (voir Monte, 2003a). Ce type d'approche m'a permis de caractériser de façon non triviale le locuteur des textes de P. Jaccottet par son usage des interrogations, des injonctions, des négations et des modalisateurs (pour un résumé voir Monte, 2003b). J'ai poursuivi la réflexion en m'intéressant aux cas inverses d'effacement énonciatif où l'énonciateur textuel présente l'énoncé comme doté d'une autorité intrinsèque détachée de son énonciation: j'ai examiné à la fois l'enracinement historique de ces types de poèmes (Monte, 2006), les difficultés qu'ils posent parfois à leur récepteur et leur argumentativité cachée (Monte, 2007). Mises à l'épreuve sur des corpus différents, ces recherches ont débouché sur deux synthèses (Monte, 2018a et à paraitre). J'y reviendrai dans l'analyse des deux poèmes.

Dans cette approche énonciative, j'ai rencontré la question de l'éthos, cette image de soi que le locuteur cherche à donner de lui-même et qui était considérée comme un des types de preuves par Aristote. Ce concept élaboré d'abord pour les discours à visée argumentative explicite a été ensuite étendu par D. Maingueneau (2002) à d'autres types de textes, notamment les textes littéraires dotés d'un ton, d'une vocalité spécifique. Ce concept m'est apparu comme particulièrement fécond pour analyser le type de relation qui s'établit entre le poème et son lecteur, invité à adhérer au monde du poème et à la voix qui le porte. Parler d'éthos c'est dire que «quelque chose de l'ordre de l'expérience sensible se joue dans le processus de la communication verbale. [...] Pris par la lecture dans un éthos enveloppant et invisible, on ne fait pas que déchiffrer des contenus, on participe du monde configuré par l'énonciation, on accède à une identité en quelque sorte incarnée » (ibid., p. 66).

On trouvera dans H. Laroche et M. Monte (2016) une réflexion générale sur l'éthos en poésie et dans M. Monte (2016b) une analyse linguistique de l'éthos chez deux poètes, J. Sacré et A. Émaz. L'éthos n'est pas à concevoir comme une figure de l'auteur dotée de caractéristiques psychologiques ou sociales mais comme une manière de dire qui est aussi une manière d'être et qui a une valeur d'appel, d'invite. Il importe en effet de distinguer entre un éthos dit - ce que le locuteur dit de lui-même, de ses affects, de son caractère et un éthos montré, indiqué par les caractéristiques mêmes du texte, ce qui pose la question des frontières entre l'éthos et le style. D. Maingueneau (2015, p. 296) conçoit le style comme le résultat de "l'intention de montrer un éthos à travers la mise en scène d'un certain investissement de la langue » qui peut aller jusqu'à "éliminer la dimension 
informative au profit du seul éthos» (ibid., p. 294). Dans le texte littéraire où l'intention "stylifiante » est presque toujours présente et où la dimension référentielle du texte est étroitement imbriquée à sa dimension esthétique, les deux notions se recouvrent partiellement. Comme je le disais dans M. Monte (2016a, p. 1873) :

[d]échiffrer un style, c'est porter un regard sur le processus de production du texte, que l'éthos envisage du point de vue de l'effet produit à réception. Le style connote en effet la volonté du scripteur, son inscription consciente dans une tradition ou sa contestation de celle-ci, et s'appréhende notamment dans l'étude de la genèse du texte (Herschberg-Pierrot 2005). L'éthos désigne, lui, l'image que le lecteur peut se faire de l'auteur grâce à un faisceau d'éléments linguistiques qui incarnent le texte. La distinction s'avère donc assez artificielle dans la mesure où, in fine, les deux concepts articulent une intentionnalité, des formes et des effets.

6 Un des points cruciaux de la notion d'éthos sur le plan théorique est la question de l'intentionnalité : celle-ci est forcément engagée dès lors qu'on est dans une pratique écrite faisant du scripteur son propre lecteur et qui plus est dans un projet littéraire qui suppose un positionnement par rapport à des modèles ou contre-modèles. Mais elle ne doit pas être entendue au sens d'une pleine maitrise de ce que l'on fait quand on écrit mais plutôt comme une recherche d'adéquation entre ce qui pousse à écrire (et qui, selon les auteurs, peut aller de la simple disponibilité à un mouvement intérieur ou à un élément extérieur jusqu'au projet le plus précisément défini) et ce qui s'écrit sur la page. C'est l'usage spécifique du langage qui est fait par l'énonciateur ainsi que la présence dans le texte de certaines thématiques et de ce que J.-B.Grize (1990) appelle des schématisations, présentant le monde référentiel d'une certaine façon, qui produisent, sans que le scripteur le contrôle vraiment, un certain effet que le récepteur peut choisir de rapporter à une image de l'énonciateur. Un tel processus n'exclut pas des malentendus, dans la mesure où, comme l'affirme P. Jousset (2015, p. 88), l'éthos est une interface : « Nous n'avons affaire qu'à des effets éthiques en littérature, ou du moins c'est eux seuls que nous pouvons apprécier. L'éthos doit être regardé plutôt comme une interface, qui établit un dialogue forcément asymétrique, amputé, ou plutôt grandement forgé, imaginaire, non pas donné mais constitué par la lecture. »

Le récepteur peut aussi refuser une telle lecture qui personnalise la relation et considérer le texte comme un objet matériel offert à sa jouissance sans construire une figure d'énonciateur. Cependant, je préfère penser que l'érection d'un corps esthétique, au sens où l'entendait G. Molinié (2005, p. 153-154) passe chez le récepteur, quoi qu'il en ait et au minimum, par « un effet de voix ou de vision, dont l'agent et la source sont escamotés, épuisés à l'intérieur de cet effet, [...] voix entendue sans présence d'un larynx, d'une gorge, d'une bouche, sans le visage de sa profération. Mais effet vécu de voix " (ibid, p. 166). Pour G. Molinié, cet effet de présence que j'appelle l'éthos est à rapporter à un actant qui n'est pas l'auteur, mais «l'idée d'auteur » (Molinié, 1998, p. 59), qui est plutôt « un lieu indéterminé [...] caractérisé comme une puissance d'excitabilité et d'émission » ( ibid.) qui n'est mue que par le pôle opposé, celui de la réception. Pour ma part, il me semble possible de le rapporter à l'énonciateur textuel construit par le lecteur en réponse à l'offrande du texte. Parler d'énonciateur textuel et non d'auteur, c'est précisément reconnaitre, avec l'analyse de discours, qu'au-delà de son individualité biographique, un sujet, lorsqu'il prend la parole, devient une voix traversée par un dialogisme constitutif ${ }^{4}$ (Authier-Revuz, 1982), qui peut, par le travail d'écriture, devenir dialogisme montré et permettre ainsi le dialogue avec le lecteur à plusieurs niveaux. 


\section{Approche textuelle et comparée}

8 Cette attention à la relation potentiellement offerte par le texte passe par une écoute de la voix du texte qui, d'une façon apparemment paradoxale mais en réalité très logique, m'a conduite à élargir progressivement mon champ d'investigation au-delà des seules composantes énonciatives du poème. Impossible, en effet, d'ignorer le rôle, dans la construction de cette voix, du rythme porté par l'interaction entre vers et syntaxe, par la disposition visuelle du poème et par les figures de répétition. Mais impossible également de ne pas inclure dans l'investigation le travail sémantique sur les isotopies, la façon dont elles s'entrelacent par le biais des figures, et dont elles se déploient au fil du texte. Il s'agit ainsi d'étudier ce que F. Rastier (1989) appelle les rythmes sémantiques et d'une certaine façon de renouer avec l'approche sémiotique telle que pouvait la mener A. J. Greimas mais en observant les transformations du texte au ras de la matérialité textuelle. Il y a là, certes, un déplacement par rapport à une focalisation exclusive sur les relations énonciatives mais cette attention aux structures me parait indispensable dans la mesure où le texte littéraire, qu'il montre ou masque sa source énonciative, persuade par sa dynamique même: l'évolution du monde référentiel qu'il met en scène passe par l'évolution de la matérialité du texte, qui affecte toutes les dimensions de la langue, incluant, pour le texte écrit, sa disposition spatiale ${ }^{5}$.

9 Cherchant à appréhender la spécificité du texte poétique sans pour autant le couper des autres textes, j'ai ainsi été amenée à partir de 2012 à proposer un modèle d'analyse des textes qui les envisage sous trois aspects :

- leur dimension sémantique, c'est-à-dire la façon dont ils construisent le monde référentiel en s'appuyant sur les interactions entre les signifiés;

- leur dimension esthésique, c'est-à-dire la façon dont ils usent consciemment des potentialités sensibles de leur matériau (sons, graphies, rythmes prosodiques) pour produire des émotions et des significations qui configurent elles aussi la perception du référent par le lecteur;

- leur dimension énonciative, c'est-à-dire la façon dont ils construisent une scène d'énonciation plus ou moins fidèle à ce qui était prévisible en fonction du contrat de communication qui les régit.

10 Si on le compare aux propositions de F. Rastier $(1989 ; 1996)$ ou à celles de J.-M. Adam (2011, p. 45), ce modèle semble réduire outrageusement la complexité textuelle et les outils nécessaires pour l'interpréter. Mais ce serait se tromper sur son objectif: les trois dimensions, que j'ai présentées plus en détail dans M. Monte (2018b), interagissent bien sûr entre elles, comme je le montrerai plus bas, mais les considérer séparément vise à comparer entre eux du point de vue pragmatique des genres de textes et de discours appartenant à des sphères sociales différentes.

Sur l'axe sémantique, je défends ainsi l'idée que les textes scientifiques et les textes poétiques partagent souvent un trait commun: la construction de représentations sémantiques qui demandent au lecteur un effort spécifique, car elles s'écartent des représentations ordinaires partagées dans divers usages quotidiens. Mais cette difficulté d'accès n'est pas due aux mêmes causes: pour ne donner qu'un exemple, ce sont les processus de désignation qui sont plus complexes dans les textes scientifiques, alors que ce sont les réseaux isotopiques, les paradoxes et le flou référentiel qui désarçonnent le lecteur de poèmes. Sur l'axe esthésique, les poèmes, les chansons et les slogans 
publicitaires partagent des traits communs, qui distinguent, par exemple, les poèmes des textes littéraires narratifs (de façon générale). Sur l'axe énonciatif, on peut distinguer les textes, à quelque catégorie de discours qu'ils appartiennent, dont la scénographie ${ }^{6}$ est en accord avec les attentes suscitées par le contrat de communication qui les régit (scène englobante) et le genre auxquels ils appartiennent (scène générique) et ceux dont la scénographie est en décalage par rapport à ces attentes ou joue avec elles. Un des charmes de Jacques le fataliste, par exemple, est que le roman montre sa fictionnalité à travers les interpellations du narrateur au lecteur au lieu de feindre la véridicité des évènements et des personnages, comme beaucoup de romans de l'époque. Les clips publicitaires les plus sophistiqués jouent souvent de ces décalages.

12 En poésie, hormis certains genres codifiés par une longue tradition - la poésie épique, la fable -, les attentes relèvent plutôt de la scène englobante: on peut retracer les évolutions de ce qu'attendait un lecteur de poésie au fil des siècles. À cet égard, le poème en prose a constitué à ses débuts une rupture tant la poésie était associée au vers. Les poèmes qui utilisent la forme de la recette de cuisine ${ }^{7}$ optent pour une scénographie en rupture avec leur scène englobante et revendiquent par là un ancrage de la poésie dans la quotidienneté, a contrario des aspirations idéalistes qui lui sont souvent attachées. Il en va de même pour les descriptions-définitions de F. Ponge. La poésie retravaille souvent des genres d'autres discours qu'elle subvertit à son profit (voir Monte, 2014). La poésie lyrique joue également sur une ambigüité quant à sa scénographie. Elle se donne en effet comme un "énoncé de réalité ", pour reprendre les termes de K. Hamburger, tout en créant des scénographies non réalistes : le locuteur s'y adresse à l'être aimé, à des morts, à des éléments naturels, à des entités abstraites, tout en invoquant parfois la présence du lecteur. L'énonciation a des traits qui l'ancrent dans l'ici-maintenant de son producteur et d'autres qui la situent dans un temps et un lieu réactualisables à chaque lecture: le poème lyrique brouille ainsi les frontières entre texte autobiographique et texte fictionnel, entre texte embrayé et débrayé (voir Monte, 2003a). La poésie partage avec les autres hypergenres littéraires (roman, théâtre) mais aussi avec les discours publicitaires ou philosophiques cette propriété d'appeler des scénographies variées non routinières, qui légitiment la parole tenue dans le poème et les points de vue qui y sont défendus. Pour apprécier pleinement ces textes, le récepteur doit être conscient de la part d'invention qui leur est constitutive, voire de comprendre que l'énonciateur a fait une œuvre à la scénographie complètement originale.

La dimension énonciative implique aussi nécessairement la prise en compte de l'interdiscours aussi bien du côté du producteur qui choisit un certain positionnement par rapport à ses devanciers que du côté du récepteur qui, pour interpréter le texte, va, consciemment ou non, le confronter à d'autres textes du même genre. C'est notamment en vertu de cette confrontation que le récepteur reconnaitra une légitimité à l'énonciateur pour énoncer ce qu'il dit comme il le dit.

14 Je voudrais montrer à présent comment je mets en œuvre ces outils pour l'étude de poèmes contemporains et comme je crois à la vertu des comparaisons, j'étudierai deux textes, un de J. Sacré et un d'A. Dreyfus. Faute de place, je me concentrerai pour chaque poème sur certains traits seulement de leur écriture. 


\title{
Un poème de James Sacré
}

\author{
Elle a les genoux rassemblés comme \\ deux museaux frais d'agneaux tout \\ le silence leur est un paradis \\ d'eau - elle a les genoux comme \\ 5 deux taupinières de terreau dans l'herbe et \\ les jardins sont des paradis d'air \\ comme une couple de mots au milieu \\ de la phrase et le langage serait \\ un paradis de poèmes les genoux fermes \\ 10 et joie comme des joues.
}

Ce texte appartient à la première section d'un ensemble de poèmes intitulé «Elle a ramassé des oiseaux pour mourir» figurant dans Coeur élégie rouge, livre publié une première fois en $1972^{8}$. La composition de cet ensemble est très rigoureuse et je montrerai dans un autre article comment elle doit être prise en compte pour une compréhension fine de chaque poème. Disons, pour faire bref, que «Elle a ramassé des oiseaux pour mourir » allie deux genres différents : le poème blason qui fait l'éloge du corps féminin et le poème pseudo-encyclopédique tel qu'ont pu le pratiquer J. Renard, F. Ponge ou R. Caillois. Il est à noter que les poèmes blasons sont en vers, alors que les poèmes « encyclopédiques » sont le plus souvent en prose. L'apparition du vers dans ces séries est concomitante d'une thématique plus lyrique (p. 93, 96) ou du lien étroit qui y est fait avec la femme qui «a/ un oiseau dans les herbes ». La présence de la prose s'inscrit dans une filiation par rapport aux trois poètes cités ci-dessus, et notamment avec F. Ponge.

Voyons donc à présent ce à quoi nous invite le poème «Elle a les genoux rassemblés comme ». La première lecture permet de dégager d'emblée un trait caractéristique, commun à tous les poèmes en vers de cette partie de Cour élégie rouge et, au-delà, à de nombreux poèmes de J. Sacré : la forte discordance entre vers et syntaxe. Les contrerejets (comme, tout, et) et les rejets (d'eau, de la phrase) séparent la préposition et son régime, le prédéterminant et son groupe nominal, le nom et son complément du nom, la conjonction et la phrase qu'elle introduit. Ces distorsions introduisent des suspens, rompent les enchainements attendus, superposent deux sémantiques, celle qui est portée par la syntaxe et celle qui est portée par la disposition en vers, ce qui entre en tension avec une autre caractéristique : la continuité affirmée du début à la fin du poème. Il n'y a qu'un point final, pas d'autre majuscule que celle à l'initiale du texte, pas de virgule qui séparerait par exemple le premier énoncé ( Elle a les genoux rassemblés comme deux museaux frais d'agneaux») et le second ("tout le silence leur est un paradis d'eau »). Lorsque la phrase reprend après le tiret, les propositions sont reliées entre elles par des et qui s'accumulent. Le poème se donne donc comme une seule coulée, mais une coulée qui, de temps en temps, s'interrompt sur des mots-grumeaux dont la charge sémantique, 
fortement mise en valeur par leur place avant le blanc, éclaire la compréhension du poème. Comme, et, une couple s'inscrivent dans une isotopie ${ }^{9} \mathrm{du} / /$ lien// que tout et les syntagmes paradis d'eau, paradis d'air, par leur référence à deux des quatre éléments - le troisième, la terre, étant abondamment représenté dans le poème - font tendre vers la // totalité//, que le mot terreau peut symboliser par la conjonction de la terre et de l'eau dans son signifiant. Par ailleurs paradis donne à cette totalité une connotation heureuse et un peu naïve que viennent confirmer des syntagmes qui évoquent les bucoliques virgiliennes : deux museaux frais d'agneaux, l'herbe, les jardins et jusqu'à la couple de mots qui suggère in absentia les bœufs attelés ${ }^{10}$. La disposition du poème impose ainsi un rythme à la fois heurté et continu et une mise en relief de mots clés, appartenant à des isotopies porteuses de la signification essentielle du texte: la circulation entre la thématique amoureuse et la thématique bucolique grâce au langage qui les relie et suscite le même bonheur. Ceci se condense dans la comparaison des genoux à « une couple de mots au milieu/ de la phrase $»^{11}$. Cette façon de parler du monde comme si c'était un langage et du poème comme un élément du monde matériel, fortement soulignée ici par les parallélismes des groupes comparatifs et des trois attributs paradis d'eau/d'air/ de poèmes, est une caractéristique prégnante chez J. Sacré et présente donc dès ce recueil qui l'a fait connaitre.

Les comparaisons nous renseignent aussi sur le positionnement littéraire de J. Sacré. Si, dans des années 1970 où tout un courant de la poésie refusait le lyrisme, le titre du livre sonne comme un manifeste, la teneur de ces comparaisons nous empêche d'assimiler trop vite ce lyrisme à un penchant vers le joli. Il s'y affirme, en effet, un gout de la sensation précise, à rebours des conventions, dans les syntagmes deux museaux frais d'agneaux et deux taupinières de terreau associés par leur place identique dans le vers et leur finale en eau et qui partagent un sème de 'surgissement' et d'humidité' tout en s'opposant sur l'axe clarté (la toison des agneaux)/obscurité (le monde obscur des taupes). La fin brusque du poème accentue les connotations présentes dans ces comparaisons : après l'asyndète qui juxtapose les genoux fermes à un paradis de poèmes, le dernier vers, asyntaxique, prédique une dernière comparaison tout entière appuyée sur la parenté des signifiants /3wa/ et / $3 \mathrm{u} /$. Le poème se termine ainsi par un raccourci où se condensent à la fois la sensualité du texte, la polysensorialité (vue et toucher, voire gout) et le jeu avec les mots.

L'érotisation du langage s'opère par le rythme qui, à la fois, entraine et suspend, comme peuvent s'interrompre ou s'accélérer les caresses, mais aussi par les chaines de phonèmes. On peut suivre le $/ 3 /$ de genoux dans jardins et langage mais c'est surtout dans le dernier vers qu'il se déploie, associant ainsi les genoux à une autre partie du corps, tout aussi ronde et double, et à la joie - au jouir - du personnage et de l'énonciateur ${ }^{12}$. Sa présence reste modeste (sept occurrences). Il en va autrement pour le /l/ et le /d/ qui, avec seize occurrences chacun, sont les consonnes les plus représentées dans le texte. Le / $1 /$, outre sa présence dans « elle a les ", qui, répété à l'attaque de tous les poèmes blasons, exemplifie l'écholalie amoureuse, se densifie aux vers 3 et 8 . Quant au /d/, il a partie liée par le «deux» avec la gémellité des genoux, mais il est fortement mis en relief par sa récurrence presque cacophonique dans " paradis d'eau » et « des paradis d'air » et par sa concentration aux vers 5 et 6 marqués aussi par la récurrence du couple de phonèmes / $\varepsilon R /$ qui réunit l'herbe et l'air. Le paradis (que redouble le mot «jardins $»^{13}$ ) devient un lieu d'échos joueurs entre langues et entre syllabes. Si ces deux vers centraux, où se concentrent l'eau, la terre et l'air, portent les redoublements phoniques à leur comble, ceux-ci sont présents, outre à la clôture, en maints passages de ce court poème : deux 
museaux frais d'agneaux, comme une couple, mots au milieu, paradis de poèmes, poèmes/fermes. Plus à distance on peut associer pour leurs voyelles toniques museaux, agneaux, terreau et mots, de même que herbe, air et fermes. Le poème est ainsi placé tout entier sous le signe du deux, associé à une tonalité presque enfantine. Alors que les longueurs syllabiques et l'organisation interne des vers semblent trop irrégulières pour produire un effet perceptible ${ }^{14}$, les chaines phoniques soutiennent puissamment l'unité du poème, tout en voyant leur impact décuplé par les distorsions mètre/syntaxe qui placent quatre fois le / $\mathrm{d} /$ et deux fois le / $/$ à l'attaque des vers. L'apparente contradiction entre ruptures et coulée du poème trouve sa raison d'être dans la priorité donnée à l'architecture sonore et à ses effets d'écho.

Il m'est impossible de développer l'analyse de l'énonciation. Je ne signalerai que quelques aspects :

- Une présence discrète du locuteur en l'absence de tout acte de langage expressif ou directif, comme si le relai était confié au signifiant (genou :je/nous, forte présence du /3/ à la fin) et au découplage du vers et de la syntaxe.

- La bémolisation du dernier énoncé verbal par le conditionnel «serait», porteuse d'une réflexivité qui bride l'effusion lyrique.

Ce que signale le conditionnel, mis en valeur par le blanc de fin de vers, c'est, me semblet-il, l'hiatus entre la comparaison et la métaphore : les genoux sont comme une couple de mots, mais s'ils étaient vraiment une couple de mots, si le corps et les mots devenaient les deux faces d'une même réalité, alors le langage serait un paradis de poèmes, mais il faut raison garder et ne pas perdre de vue le «comme», l'espace de séparation. Le conditionnel vient amortir la griserie du langage au moment où, pourtant, elle s'affirme le plus fortement avec cet envol vers le paradis des poèmes. On a là un mouvement que l'on retrouve fréquemment chez J. Sacré, de confiance dans le langage doublée d'une certaine autodérision, de dilatation puis de retrait qui évoque, bien sûr, la relation érotique, mais sur un mode joueur et retenu.

21 Au terme de ce parcours, que retenir en termes de méthode? Mon entrée dans le poème s'est faite par l'attention au découpage en vers et à ce qu'il induisait comme ruptures rythmiques et mise en relief de certains mots ou syntagmes qui, pris dans des parallélismes à la fois sémantiques et phoniques, se sont révélés porteurs d'enjeux interprétatifs décisifs. L'étude énonciative, écourtée ici, enrichit la perception de l'éthos de l'énonciateur qui s'était amorcée lors de l'étude du rythme et de la dimension sémantique et permet de mieux définir le positionnement littéraire affirmé dans ce poème. 


\title{
Un poème d'Ariane Dreyfus ${ }^{15}$
}

\author{
LE FÉMININ \\ (EN RÉSISTANT) \\ à Jafar Panahi \\ cinéaste lumineux \\ Le noir est puissant et tranchant \\ Le front barré par le tchador \\ Elle marche vraiment vite \\ Et le cœur peut se tordre assez \\ 5 Pour commander aux jambes et aux pieds, \\ A elle toute qui s'assoit brusquement \\ Dans la petite cour. \\ Pour le souvenir, \\ Soleil s'appuie sur un mur. \\ 10 Devant lui, \\ Elle plonge le bras dans l'eau langoureuse. \\ Que croyez-vous? \\ Cela, aussi. \\ Pas un jour sans une rencontre \\ 15 Gardée comme l'humidité sur le tissu. \\ Le monde on verra, il recule aussi peu que les yeux des filles.
}

Ce poème, écrit en deux campagnes en 2007 et 2008, fait partie de la section « Le cadre ne casse pas » de La terre voudrait recommencer, publié en 2010. Je me propose d'y entrer par la dimension énonciative. L'énonciatrice l'inscrit dans une certaine actualité par la dédicace à un cinéaste iranien (qui a été contraint plus tard de tourner clandestinement), par le titre et le choix du mot «tchador» qui, immédiatement, évoque la situation des femmes dans la république islamique iranienne. Le poème contient une interpellation à un destinataire non précisé, qui peut être le lecteur mais aussi les adversaires de la liberté des femmes. La question « Que croyez-vous? " s'utilise souvent, en effet, pour contester les attentes de quelqu'un par rapport à une situation ${ }^{16}$. La réponse pousse à inscrire le geste de plonger les bras dans l'eau (cela) comme appartenant à une série déjà exemplifiée dans ce qui précède. La négation du vers 14 possède aussi une tonalité polémique ${ }^{17}$. Le dernier vers invite une communauté large, désignée par on, à vérifier de ses yeux le jugement émis par l'énonciatrice sur le monde ${ }^{18}$. Ce jugement contient un présupposé qui ne reprend pas un posé antérieur : « les yeux des filles ne reculent pas ». On peut donc en déduire que c'est tout le poème qui a affirmé cela. Contrairement au poème de J. Sacré, celui-ci contient un certain nombre de lexèmes évaluatifs qui montrent que la réalité est vue dans le prisme d'une observatrice: "Le noir est puissant et tranchant ", "Elle marche vraiment vite ", "Et le cœur peut se tordre assez/ pour...", "qui s'assoit brusquement ", «l'eau langoureuse ». On peut conclure de toutes ces observations que, malgré l'absence de marques de première personne, l'énonciatrice exprime son point de vue et que celui-ci est en empathie avec l'expérience de la femme désignée par « elle » ${ }^{19}$.

L'interprétation de cette expérience par le lecteur relève de la dimension sémantique. On observe tout d'abord une isotopie ${ }^{20} \mathrm{du}$ corps et des gestes qui contribue à la cohérence référentielle mais aussi à la dimension symbolique du poème : l'oppression des femmes se 
marque dans « le front barré par le tchador ", leur résistance dans l'énergie qui les anime, dont le cœur qui se tord est la métonymie, leur malice dans la sensualité dont elles investissent les gestes anodins décrits dans les vers 10-15. Le poème ne dit pas directement l'enfermement et le combat, il les indique obliquement et du coup, chaque détail se trouve investi d'un sens second. L'isotopie de la puissance et de la détermination change ainsi de camp du début à la fin du texte : attribuée au noir, réinterprété dès le vers 2 (et même avant si on tient compte de la dédicace) comme la couleur du tchador, elle passe au « elle » dans les vers 4-7, 10-11, et s'élargit à toutes les filles au vers 16. Mais on peut aussi dégager une isotopie de l'obscurité et de la lumière qui est à peine esquissée et une isotopie de l'eau, très fugace, qui semblent contenir toutes deux une forte portée symbolique. Le sème 'lumière' est présent dans lumineux, soleil, yeux et par afférence aux vers 10-11 (le soleil éclaire l'eau et la peau); il s'oppose au sème 'obscurité' porté par noir et tchador. Tout au long du texte, le sème 'lumière' concerne des personnes, et même lorsqu'il s'agit du soleil, celui-ci est personnifié à la fois par son attitude et par l'absence d'article ${ }^{21}$ : A. Dreyfus dit que, dans son poème, le soleil est « une sorte de double du cinéaste à plusieurs niveaux » mais que cela n'a émergé que progressivement. Dans la première version du poème, le lexème apparait dans l'énoncé «Encore recouverte elle bouge, elle va au soleil / Qui la dessine plus qu'il la touche »: le symbolisme est plus appuyé mais la personnification peut être entendue dans un sens faible, rhétorique, qui n'attribue pas de volonté au soleil. Ce n'est qu'après quatre ou cinq versions que la forme définitive, plus allusive mais résolument anthropomorphique, est obtenue. Les vers 8-9 jouent un rôle essentiel de charnière, ce qu'indique leur isolement par des blancs: le soleil y relie le passé au présent en s'immisçant dans l'espace domestique. À partir du vers 10 , commence la partie plus dialogique du texte où la résistance devient plus active. Un lecteur qui ignorerait tout de J. Panahi et n'aurait pas accès à l'article d'A. Dreyfus pourrait-il comprendre ce rôle dévolu au soleil ? Il me semble que oui s'il était attentif à l'enchainement du texte: le syntagme «devant lui » indique en effet clairement que la femme accomplit son geste en étant consciente de la présence du soleil. La présence de langoureuse ${ }^{22}$, de rencontre, l'évocation du toucher dans le vers 15 et le contexte énonciatif polémique orientent assez nettement vers une interprétation du soleil comme allégorie du masculin source de désir, ce que facilite l'absence de déterminant et l'anaphore en lui.

L'évolution des figures mobilisées par le texte est aussi significative de sa dynamique. En effet, alors que le début du poème est marqué par des métonymies et synecdoques qui permettent une approche sensorielle de la réalité mais ne la transforment pas radicalement, la fin contient une hypallage ( (')eau langoureuse »), une sorte de syllepse sur lui (à la fois soleil et homme), un euphémisme (rencontre) adossé à une comparaison qui s'appuie elle-même sur une métalepse (l'humidité pouvant être rapportée à sa cause, eau sur le vêtement, mais aussi, allusivement, sueur ou sécrétions sexuelles). Ces figures d'indirection, sans perdre l'ancrage corporel, font écho à la capacité de double jeu des femmes qu'elles reproduisent dans la langue.

La dimension esthésique, quant à elle, vient ici en appui aux dimensions énonciative et sémantique. Je ne peux malheureusement l'étudier en détail et ne mentionnerai que quelques traits saillants :

- le découpage en laisses ${ }^{23}$ souligne l'opposition entre le vers 1 et vers 16 , sépare la marche et l'arrivée dans la maison (laisses 2 et 3 d'abord réunies dans une première version), donne une place centrale au soleil. Contrairement au poème de J. Sacré, qui jouait sur la distorsion vers/syntaxe, ici les vers correspondent à des syntagmes complets, voire à des énoncés 
entiers. De ce fait, le dernier vers se distingue à la fois par sa longueur exceptionnelle et par le fait qu'il rassemble deux énoncés, montrant ainsi la solidarité entre le monde (complice de ce désir d'émancipation) et les yeux des filles ;

- la mise en relief des adverbes assez et brusquement et le rejet de gardée qui confère au souvenir la dimension concrète d'un trésor ou d'un secret ;

- le tissage sonore plus serré de la deuxième moitié du texte: allitération en $/ \mathrm{s} /$, forte présence $\mathrm{du} / \mathrm{i} /$ souvent sous l'accent de fin de vers, présence doublée du /l/ et dans des mots pleins qui disent la sensualité et le désir ;

- changement de contexte des nasales /ã/ et /õ/ associées au noir et au tchador dans la première partie, à la sensualité dans la deuxième (vers 10-11, 14), qui renoue avec le «en résistant » du titre.

Ces récurrences phoniques donnent à cette partie une unité plus forte, et procurent un plaisir à l'oreille qui peut préfigurer la victoire des femmes. Ma démarche, on le voit, a consisté à repérer tout d'abord les marques d'engagement (dimension énonciative) puis à analyser la construction sémantique qui inscrit l'engagement non pas dans une dénonciation explicite mais dans la progression même du poème. Le fait de pouvoir s'appuyer sur des états antérieurs du texte montre bien que l'écrivaine a travaillé dans le sens d'une indirection plus forte, reposant sur des ellipses et sur le choix d'un vocabulaire et d'une disposition déclencheurs d'inférences. Mais l'étude attentive du texte donne accès à ce choix stylistique même si l'on ne dispose pas de ces premières versions.

\section{Conclusion}

La confrontation des deux analyses permet de voir que ma démarche part de ce qui est le plus évident à première lecture mais qui met sur la voie d'éléments moins immédiats : de la distorsion vers/syntaxe chez J. Sacré aux isotopies puis aux chaines phoniques, de l'engagement énonciatif chez A. Dreyfus aux isotopies et à la structure visuelle et sonore du poème. Elle balaie ensuite les trois dimensions que j'ai évoquées en première partie en essayant de dégager les propositions de sens présentes dans le poème à tous les niveaux de sa structure linguistique, aussi bien dans ce qu'il dit que dans ce qu'il exemplifie ou suggère. Ce faisant, on peut mesurer sur quel plan le poème se distingue ou se rapproche d'autres productions de la même époque, aussi bien dans le champ poétique que dans l'ensemble des discours ${ }^{24}$. L'analyse des deux textes a ainsi permis de dégager des traits généraux propres à de nombreux poèmes contemporains - des contenus sémantiques qui ne se révèlent que par la mise en relation de différentes structures, un ancrage fort dans l'expérience corporelle -, tout en montrant que la dimension esthésique peut être beaucoup plus forte dans certains poèmes que dans d'autres. La démarche permet ainsi de comparer les poèmes et, peut-être, de comprendre ce qui, dans chacun, va être une porte d'accès pour tel lecteur et un obstacle pour tel autre : l'un entrera dans le poème par la dimension esthésique, alors que l'autre sera sensible au premier chef à la dimension énonciative et un troisième à la dimension référentielle. Cette analyse n'est pas incompatible avec la théorie de l'évocation proposée par M. Dominicy (2011) qui s'attache, pour sa part, à préciser comment la double organisation du poème linguistique et poétique - suscite chez le lecteur un processus évocatif spécifique. Mais il est vrai que la question que je me pose est plutôt celle-ci : sur quels éléments de ce texte puis-je m'appuyer pour l'interpréter? Au fil de l'étude, je rencontre nécessairement des aspects qui ressortissent à l'évocation mais mon objectif principal reste de rendre 
intelligible des textes souvent jugés énigmatiques par maint lecteur en montrant comment l'attention à leurs différentes dimensions peut permettre d'éviter des erreurs d'interprétation dues à la non prise en compte de certains éléments ou à une focalisation excessive sur d'autres. Il appartient maintenant au lecteur d'évaluer la fécondité et la reproductibilité de ma démarche.

\section{BIBLIOGRAPHIE}

ADAM, J.-M. (2011). La linguistique textuelle. Paris : A. Colin.

ADAM, J.-M. (2018). Le paragraphe. Entre phrases et texte. Paris : A. Colin.

ANSCOMBRE, J.-C. \& DUCROT, O. (1983). L'argumentation dans la langue. Bruxelles : P. Mardaga.

AUTHIER-REVUZ, J. (1982). « Hétérogénéité montrée et hétérogénéité constitutive : éléments pour une approche de l'autre dans le discours ». DRALV 26, p. 91-151.

Dominicy, M. (2011). Poétique de l'évocation. Paris : Classiques Garnier.

DREYFUS, A. (2010). La terre voudrait recommencer. Paris : Flammarion.

FAVRIAUD, M. (2014). Le plurisystème ponctuationnel français à l'épreuve de la poésie contemporaine. Limoges : Lambert-Lucas.

GRIZE, J.-B. (1990). Logique et langage. Gap : Ophrys.

HAMBURGER, K. (1986) [1977]. Logique des genres littéraires. Trad. de l'allemand par P. Cadiot. Paris : Éd. Le Seuil.

HERSCHBERG-PIERROT, A. (2005). Le style en mouvement. Littérature et art. Paris : Belin.

JAKOBSON, R. \& LÉVI-STRAuSS, C. (1962). « “Les Chats” de Baudelaire ». L'Homme 2 (1), p. 5-21. En

ligne : https://www.persee.fr/doc/hom_0439-4216_1962_num_2_1_366446.

JousSET, P. (2015). « Autour de la notion d'éthos ». In : Jousset, P. (dir.), L'homme dans le style et réciproquement. Aix-en-Provence : Presses universitaires de Provence, p. 83-92.

LAROCHE, H. \& MONTE, M. (2016). « La fabrique de l'éthos : idéal de transparence et travail du style ». Babel. Littératures plurielles 34, p. 9-17. En ligne : https://journals.openedition.org/ babel/4575.

MAINGUENEAU, D. (2002). « Problèmes d'ethos ». Pratiques 113, p. 55-67. En ligne : https:// www.persee.fr/doc/prati_0338-2389_2002_num_113_1_1945.

MAINGUENEAU, D. (2004). Le discours littéraire. Paratopie et scène d'énonciation. Paris : A. Colin. MAINGUENEAU, D. (2015). « De l'ethos au style : la présentation de soi sur les sites de rencontre ». In : Bordas, É. \& Molinié, G. (éds.), Style, langue et société. Paris : H. Champion, p. 283-298. MoLINIÉ, G. (1998). Sémiostylistique. L'effet de l'art. Paris : Presses universitaires de France. MOLINIÉ, G. (2005). Hermès mutilé. Paris : H. Champion. 
MONTE, M. (2003a). « Essai de définition d'une énonciation lyrique. L'exemple de Philippe Jaccottet ». Poétique 134, p. 159-181. En ligne : https://www.cairn.info/revue-poetique-2003-2page-159.htm.

MONTE, M. (2003b). « Entre refus des illusions et ouverture à l'innommé : l'appel têtu de Philippe Jaccottet ». Méthode! 5, p. 205-216.

MONTE, M. (2006). « Runes de Jean Grosjean et La grande neige d'Yves Bonnefoy : de l'étrangeté pragmatique à la lecture allégorique ». In : Brophy, M. \& Gallagher, M. (éds), Sens et présence du sujet poétique. La poésie de la France et du monde francophone depuis 1980. Amsterdam/New York : Rodopi, p. 227-241.

MONTE, M. (2007). « Poésie et effacement énonciatif ». Semen 24, p. 99-121. En ligne : https:// journals.openedition.org/semen/6113.

MONTE, M. (2014), « Sonnets d'Yves Bonnefoy, Valérie Rouzeau et Robert Marteau : plan de texte et généricité ». Congrès mondial de linguistique française, p. 2835-2850. En ligne : https://www.shsconferences.org/articles/shsconf/pdf/2014/05/shsconf_cmlf14_01065.pdf.

MONTE, M. (2016a). « De l'éthos, du style et du point de vue en poésie ». In : Colas-Blaise, M., Perrin, L. \& Tore, G. M. (dirs), L'énonciation aujourd'hui. Un concept clé des sciences du langage. Limoges : Lambert-Lucas, p. 179-200.

MONTE, M. (2016b), «L'éthos en poésie contemporaine : le cas de James Sacré et d'Antoine Émaz ». Babel. Littératures plurielles 34, p. 257-281. En ligne : https://journals.openedition.org/babel/4691.

MONTE, M. (2018a). « La dimension argumentative dans les textes poétiques : marques formelles et enjeux de lecture ». Argumentation et analyse du discours 20. En ligne : https:// journals.openedition.org/aad/2511.

MONTE, M. (2018b). «Interpréter le poème : une interaction variable entre trois dimensions textuelles (sémantique, esthésique et énonciative)». In : Achard-Bayle, G. et al. (éds), Les sciences du langage et la question de l'interprétation (aujourd'hui). Limoges : Lambert-Lucas, p. 127-154.

MONTE, M. (à paraitre). « Les relations entre énonciateur textuel, locuteurs et personnages dans quelques scénographies poétiques : José-Flore Tappy, Eugène Guillevic, Jacques-Henri Michot ». In : Biglari, A. \& Watteyne, N. (dirs), Scènes d'énonciation de la poésie lyrique moderne. Paris : Classiques Garnier.

RABATEL, A. (1998). La construction textuelle du point de vue. Lausanne : Delachaux \& Niestlé.

RABATEL, A. (2017). Pour une lecture linguistique et critique des médias. Empathie, éthique, point(s) de vue . Limoges : Lambert-Lucas.

RASTIER, F. (1984). «Ah! Tonnerre! Quel trou dans la blanquette! Essai de sémantique interprétative ». Langue française 61, p. 27-54. En ligne : https://www.persee.fr/doc/ lfr_0023-8368_1984_num_61_1_5181.

RASTIER, F. (1987). Sémantique interprétative. Paris : Presses universitaires de France.

RASTIER, F. (1989). Sens et textualité. Paris : Hachette.

RASTIER, F. (1996). « La sémantique des textes : concepts et applications ». Texto! 11 (1). En ligne : http://www.revue-texto.net/Inedits/Rastier/Rastier_Concepts.html\#2.2.

RIFFATERRE, M. (1971) [1966]. « La description des structures poétiques : deux approches du poème Les Chats de Baudelaire ». In : Riffaterre, M., Essais de stylistique structurale. Trad. par D. Delas. Paris : Flammarion, p. 307-364. 
SACRÉ, J. (2001) [1972]. Cour élégie rouge. Marseille : A. Dimanche.

\section{NOTES}

1. Je remercie M. Favriaud pour ses commentaires sur la première version de ce texte.

2. L'énonciateur textuel est l'auteur impliqué : dans un poème à la première personne, il fait souvent corps avec le locuteur, à l'instar de ce qui se produit dans l'autobiographie, mais il peut aussi s'en distancier ironiquement (voir Monte, à paraitre).

3. On trouvera dans cet article une réflexion approfondie sur l'usage et les implications des deux notions.

4. Ce serait ainsi traduire dans des mots modernes et théoriques ce qu'entendaient les classiques par la Muse, ou l'inspiration.

5. On trouvera des analyses très fines sur la ponctuation dans M. Favriaud (2014) et sur le paragraphe dans J.-M. Adam (2018).

6. Sur les trois scènes qui, selon D. Maingueneau, composent la scène d'énonciation, on peut lire un résumé dans D. Maingueneau (2002, p.64) et une étude approfondie appliquée au discours littéraire au chapitre 17 de D. Maingueneau (2004).

7. « Recette » d'E. Guillevic, « Pour un art poétique » de R. Queneau.

8. Les références aux pages se font dans l'édition de 2001 donnée en bibliographie.

9. Sur les concepts d'isotopie et de sèmes inhérents vs afférents, voir F. Rastier (1987). Sur la façon dont on peut s'appuyer sur le concept d'isotopie pour un travail avec les élèves, voir F. Rastier (1984).

10. Dans le dialecte poitevin-saintongeais, une couple signifie "plusieurs, un petit nombre » (je remercie M. Favriaud pour cette information). J. Sacré joue sur les sens du mot masculin et du mot féminin.

11. Il y a théoriquement une ambigüité syntaxique pour l'incidence du groupe comparatif, mais le rattacher aux jardins parait moins vraisemblable compte tenu de la logique d'ensemble du texte. On pourrait ponctuer la phrase ainsi : «elle a les genoux comme deux taupinières de terreau dans l'herbe (et les jardins sont des paradis d'air), comme une couple de mots au milieu de la phrase (et le langage serait un paradis de poèmes), les genoux fermes et joie comme des joues. »

12. Confronter le poème à l'ensemble de l'œuvre permettrait de déplier certains implicites de ce texte, moins irénique qu'il n'y parait (les genoux serrés peuvent être une protection contre le viol).

13. Le mot hébreu employé dans la Genèse (II, 8) pour le jardin planté par Dieu en Éden a été traduit en grec par paradeisos, terme d'origine perse désignant un jardin royal ou mystique avec eau.

14. Le nombre de syllabes est le suivant : $10-7-10-7-11-9-10-10-12-6$, et les vers ne possèdent pas d'organisation interne récurrente.

15. Un commentaire d'A. Dreyfus sur le chantier d'écriture de ce texte se trouve sur le site Poezibao: http://poezibao.typepad.com/poezibao/2009/05/un-chantier-de-po\%C3\%A8me-parariane-dreyfus.html.

16. M. Favriaud me signale que cette formule est courante en persan et figure peut-être aussi dans la traduction sous-titrée de Hors jeu, qui a inspiré A. Dreyfus.

17. Sur les négations polémiques, on pourra lire J.-C. Anscombre et O. Ducrot (1983).

18. L'expression « on verra bien » se trouve ici resémantisée.

19. Pour une analyse de la façon dont le discours associe empathie et point(s) de vue, lire

A. Rabatel (2017, p. 299-314). 
20. Si l'espace qui m'est imparti le permettait, je montrerais comment les isotopies évoquées dans ce paragraphe sont construites par le lecteur, quels morphèmes ou syntagmes les actualisent. Ne pouvant le faire, je ne recours pas à la notation entre barres obliques.

21. A. Dreyfus écrit dans Poezibao: «[...] en passant de «Le soleil » à «Soleil », j’ai la joie d'arriver enfin à faire de lui un être quasi masculin, et donc vraiment rencontré malgré les interdits!»

22. A. Dreyfus dit que ce mot ne fait pas partie de son vocabulaire, qu'il s'est imposé à elle. Il s'agit peut-être d'une réminiscence de la première ariette de Verlaine dans Romances sans paroles «C'est l'extase langoureuse/ C'est la fatigue amoureuse ».

23. J'appelle ainsi des paquets de vers en poésie non métrique, réservant à la poésie métrique l'utilisation du mot strophe.

24. L'appui sur des bases de textes numérisés et l'amélioration constante des logiciels d'exploration outillée dont nous disposons devraient permettre pour cette comparaison de ne plus dépendre uniquement de la connaissance nécessairement partielle de l'analyste.

\section{RÉSUMÉS}

Cet article défend une approche linguistique de la poésie contemporaine qui s'appuie à la fois sur les données énonciatives, les parcours sémantiques reposant sur les isotopies et les faits de disposition, de rythme et de chaines phoniques. Il met la démarche en œuvre à propos d'un poème de J. Sacré et d'un poème d'A. Dreyfus en montrant comme un lecteur armé d'outils linguistiques simples mais robustes peut construire l'interprétation du texte en tenant compte de l'interrelation entre les dimensions sémantique, énonciative et esthésique. Ce faisant le lecteur peut aussi confronter les choix opérés dans le poème à l'état du champ poétique à un moment donné et analyser la scène d'énonciation et l'éthos construits par le texte.

In this paper we define a linguistic approach to contemporary poetry that focuses on the speaker's marks of subjectivity, on textual structure, rhythm and phonic chains and on semantic paths based on isotopies. We carry out this approach in the study of two poems, one from J. Sacré, the other from A. Dreyfus and show how simple but solid linguistic tools allow the reader to construe the interpretation of the text taking into account the interrelation between semantic, enunciative and aesthesic dimensions. In doing so the reader can also compare the devices observed in the poem with the setting of the poetic field at a given moment and analyse the implied author's position and ethos displayed in the text.

\section{INDEX}

Mots-clés : sémantique textuelle, isotopie, énonciation poétique, dimension esthétique du poème, rythme, éthos, poésie française des XXe et XXIe siècles

Keywords : textual semantics, isotopy, poetic enunciation, aesthesic dimension of the poem, rhythm, ethos, 20th and 21st century-French poetry 


\section{AUTEUR}

\section{MICHĖLE MONTE}

Université de Toulon, Babel, EA 2649, F-83000, France 